\title{
Comparison of Shearography to Scanning Laser Vibrometry as Methods for Local Stiffness Identification of Beams
}

\author{
F. Zastavnik*, L. Pyl*, J. Gu*, H. Sol*, M. Kersemans ${ }^{\dagger}$ and W. Van Paepegem ${ }^{\dagger}$ \\ *Department of Mechanics of Materials and Constructions (MeMC), Vrije Universiteit Brussel (VUB), Pleinlaan 2, I050, Brussels, Belgium \\ †Department of Materials Science and Engineering, Universiteit Gent (UGent), Technologiepark-Zwijnaarde 903, 9052, Zwijnaarde, Belgium
}

\begin{abstract}
Local stiffness of Euler-Bernoulli beams can be identified by dividing the bending moment of a deformed beam by the local curvature. Curvature and moment distributions can be derived from the modal shape of a beam vibrating at resonance. In this article, the modal shape of test beams is measured by both scanning laser vibrometry (SLV) and shearography. Shearography is an interferometric optical method that produces full-field displacement gradients of the inspected surface. Curvature can be obtained by two steps of derivation of the modal amplitude (in the case of SLV) or one step of derivation of the modal shape slope (in the case of shearography). Three specially prepared aluminium beams with a known stiffness distribution are used for the validation of both techniques. The uncertainty of the identified stiffness distributions with both techniques is compared and related to their signal-to-noise ratios. A strength and weakness overview at the end of the article reveals that the shearography is the technique that shows the most advantages.
\end{abstract}

KEY WORDS: Euler-Bernoulli beams, scanning laser vibrometry, shearography, stiffness identification

\section{Introduction}

Data collection for local stiffness identification of beams can be divided into dynamic-based and static-based methods. The literature and applications of static-response-based identification is relatively limited. Most authors compute the curvature of test beams from measured displacements. The displacements under a known static loading are curve fitted or directly compared with numerical models [1-4]. Yang et al. [5] and Li et al. [6] used inverse procedures for reconstructing the stiffness distribution in beams using static responses. The deflection profile was obtained by processing digital images of the beam and was next utilised as the input for inverse computational procedures. An inverse method based on a finite element model and an optically measured displacement field was used by Sztefek and Olsson [7]. Grédiac et al. used full-field techniques in combination with the virtual field method to evaluate distributed material stiffness [8]. Devivier et al. used full-field slope measurements in combination with the virtual field method to evaluate stiffness and damage in composite material plates [9].

The literature on dynamic methods for the identification of distributed stiffness is much more extended. Dynamic methods use characteristics such as natural frequencies [10], displacement modal shapes [11], modal shape derivatives $[12,13]$, wavelet analysis of dynamic signals [14] and harmonic responses [15, 16]. An overview of dynamic-response-based methods using inverse techniques can be found in [17]. An important application of stiffness identification in beams, plates and shells is the analysis of damage profiles. It is accepted by many authors that local stiffness is a good quantity to monitor local damage. Damage accumulation in structures can be associated to stiffness reduction and expressed mathematically as a stiffness factor or a damage variable related to the stiffness [18-21]. The induced damage can be translated into a modification of structural mass, damping and stiffness. A vast amount of methods exists that examine changes in measured vibration response to detect, locate and characterise damage in structural systems. The basic idea behind these methods is that natural vibration parameters (notably natural frequencies, modal shapes and modal damping ratios) are functions of the physical properties of the structure (mass, damping and stiffness). Therefore, changes in the physical properties will cause detectable changes in the modal properties. Literature overview of damage identification methods using vibration analysis is given, among others, by Doebling et al. [18] and Farrar et al. [19-21].

It is a well-known fact that differentiation of measured data can lead to noisy results. The computation of curvatures from measured modal shapes requires the second derivative, hence, two steps of differentiation. In all the methods found in the previously cited literature using curvatures, the measured modal shapes or modal shape slopes are first curve fitted with polynomial expressions to overcome the errors due to differentiation. Curve fitting, however, is not straight forward: curve fitting with too low order polynomials destroys the useful information and curve fitting with too high order polynomials tends to fit the noise as well. The optical technique shearography (described later in the text) offers the advantage that modal shape slopes are measured instead of modal shapes. The computation of the curvature hence requires only a single step of differentiation.

This paper describes a method for identifying the local stiffness distribution of beams by measuring the free-free modal bending shape associated with the first natural 
frequency. Thin beam Euler-Bernoulli behaviour is assumed. The local bending moments are divided by the local curvatures to yield the local beam stiffness. In the test set-up, the beams are freely suspended by thin wires and are acoustically excited.

The performance of the proposed identification method is validated with experiments on aluminium beams with a known stiffness distribution. Different beam shapes with respect to the thickness distribution are tested: flat, concave and convex specimens.

Two different optical methods are used for data acquisition: scanning laser vibrometry (SLV) and shearography. The SLV yields the modal shape, and shearography yields the gradient (slope) of the first modal shape of test beams. In both cases, the measured values are curve fitted with a high-degree polynomial. Curve fitting enables differentiation and is also used to estimate the noise level, as will be explained later in this paper.

Shearography $[22,23]$ is an interferometric full-field imaging technique that can produce the gradient of deformation of the inspected surface. From its conception [24, 25], it was envisioned as a quantitative technique to determine strain, but presently, it is mostly used as a qualitative technique, to visualise defects in material [26]. It is used in health monitoring of aircraft structures [27-29], for inspection of bonding quality in joints [30] and for reinforcement of concrete beams [31]. Shearography has become widely used for defect detection in the pneumatic tire industry [32]. Visual inspection can be enhanced to numerically determine the size and depth of a defect [33].

Shearography can be used quantitatively, to measure the surface strain directly [22]. Depending on the optical arrangement of the light source(s) and the camera(s), shearography can be made sensitive to out-of-plane or in-plane deformation [23], and three of six independent components of the strain tensor can be measured directly. However, in this paper, only the out-of-plane deformation gradient was sufficient to characterise the beam stiffness. Vibrational analysis is also possible, through the use of stroboscopic illumination [34]. An error analysis of shearography as a quantitative tool has been done by Goto and Groves [35, 36].

Shearography falls into a group of modern optical full-field methods for strain evaluation, which includes deflectometry $[37,38]$, digital image correlation (DIC) [39] and electronic speckle pattern interferometry (ESPI) [40]. Deflectometry uses a rectangular grid projected on the surface of a test object and observed with a charge-coupled device (CCD) camera. By comparing the grid in a deformed position with a reference (undeformed) position, the deformation gradients of the surface can be captured by the shift of the grid and used for stiffness identification. Shearography is derived from ESPI [25]. The main difference between shearography and ESPI is that ESPI output is related to the deformation, while shearography output is related to the gradient of deformation. ESPI is, however, much more sensitive to outside disturbances. DIC is not an interferometric but a purely optical technique, thus viable for a different scale of deformations. While shearography and ESPI operate at micrometre scale deformations, DIC is most often used at 0.1-10 mm deformation scales [41].

The configuration used in this paper is sensitive to outof-plane deformations. The vibrating beams are placed so that their vibrations occur as out-of-plane deformations. The vibrating beams are illuminated by stroboscopic illumination, allowing shearography to image only a certain phase of the modal shape. This way, the gradient of deformation is the slope of the amplitude of the modal shape. The shearography system used is the isi-sys SE3 [42].

Scanning laser vibrometry uses a directed laser beam to measure the vibrational response of a point on a surface. With the implementation of a scanning head, it has the ability to consecutively scan points, thus creating a twodimensional distribution of vibrations. SLV system Polytec PSV-400 is used for data acquisition.

The first section of this paper describes the principles of shearography. The second section describes briefly the principles of SLV. In the third section, the principle of identifying the beam stiffness from the measured modal shapes is illustrated. The next sections present the validation procedure on the aluminium beams. It will be shown that the information contents of only the first bending modal shape is sufficient for the identification of the local stiffness between 25\% and 75\% (the central 50\%) of the beam length.

\section{Shearography}

A schematic of the shearography procedure that acquires the out-of-plane gradient of deformation is presented in Figure 1. This is the set-up used for measurements in this paper. The points $A, B$ and $C$, lying on the surface of the observed object, are illuminated by coherent laser light. The light from two points $-A_{1}$ and $C_{1}-$ on the surface of the inspected object is guided by the shearing device onto the same point (pixel at $\{i, j\}$ ) of the CCD sensor. The two points $A_{1}$ and $C_{1}$ are separated by a shear distance in the $x$-direction, $\delta x$. This shearing distance is controlled by the shearing device and can be set to be in either $x$-direction or $y$-direction, or both. Depending on the shearing setting, a different gradient of out-of-plane deformation is acquired. In this paper, shearing is set only in the $x$-direction, so the acquired data are only the gradient of deformation $\partial W / \partial x$, with $W$ representing the deformation in the $z$-direction.

In the state 1 , which represents the undeformed state, the two light waves coming from $A_{1}$ and $C_{1}$ are in phase. The light waves will constructively interfere and will register as a maximum intensity on the CCD. The intensity captured 


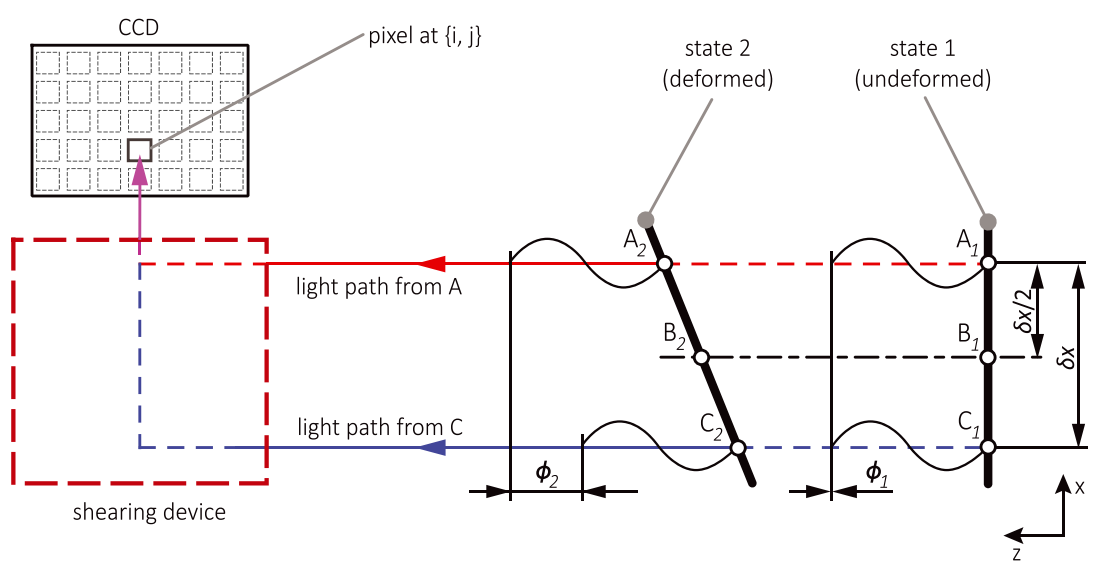

Figure I: Deformation of the observed surface creates a phase difference, which is registered as an intensity difference on the CCD sensor

by the CCD camera can be evaluated [22] into the difference of phases of the two light waves, $\varphi$. For the undeformed state, as illustrated in Figure 1, the phase difference will be evaluated to $\varphi_{1}=0$ (in radians). When the surface is deformed - state 2 - two points $A_{2}$ and $C_{2}$ are out of phase, and the CCD at that pixel will register a smaller intensity. The phase difference between $A_{2}$ and $C_{2}$ is $\varphi_{2}$. The relative phase change (in radians) is defined as $\Delta=\varphi_{2}-\varphi_{1}$ and encodes the gradient of deformation.

The gradient of deformation is calculated from relative displacements of points $A$ and $C$ and is valid for the point $B$. In the $1 \mathrm{D}$ case presented in Figure 1 , where $W$ is the displacement in the $z$-direction, the displacement gradient $\partial W / \partial x$ of a point on the surface at $\{x\}$ will be encoded by the relative phase change of its neighbouring points $\{x+\delta x / 2\}$ and $\{x-\delta x / 2\}$. The whole surface of the inspected object is registered as a $\Delta$-image with each point at pixel $\{i, j\}$ encoding the local gradient of deformation. In order for the point to have its gradient of deformation properly captured, it needs to have points $\{x+\delta x / 2\}$ and $\{x-\delta x / 2\}$ on the same surface. The edges of the scanned surface in the region $(0, \delta x / 2)$ do not satisfy this condition, so in the $\Delta$-image, they are not captured. In this paper, the missing gradient values at the edges are estimated by linear extrapolation.

The shearing distance, $\delta x$, is variable and can be set to a desired value. As the shearing distance $\delta x$ is increased, keeping the deformation constant, $\Delta$ is increased, effectively increasing the sensitivity to deformations. The values of $\Delta$ are relative to the wavelength of the used light, $\lambda$, so the value of the displacement difference will be $\lambda \Delta / 2 \pi$. The gradient of deformation can be calculated as [22]

$\frac{\partial W}{\partial x}=\frac{\lambda \Delta}{2 \pi k_{z} \delta x}$

where $k_{z}$ is the sensitivity vector in the range $(0,2)$, dependent on the positions of the light source and the camera, relative to the measured surface. Both the sensitivity vector $k_{z}$ and the shearing distance $\delta x$ can have locally varying values, which can influence the accuracy of the measurement. In this paper, they are assumed to be constant as they do not influence the noise in the produced $\Delta$-image. The locally varying sensitivity $k_{z}$ and the shearing distance $\delta x$ will be addressed in future papers, similar to [35] and [36]. Equation (1) is valid for small $\delta x$.

To acquire deformation gradients from harmonically vibrating objects, stroboscopic illumination can be used [34]. When the light pulses are synchronised with the excitation signal, only the vibrations at a fixed vibration phase are observed. This effectively freezes the vibration and enables modal shape slope acquisition for the beams vibrating at the first resonant frequency. The measured gradient of deformation $\partial W / \partial x$ will thus be the slope of the measured first modal shape.

The images that shearography is producing have a certain amount of noise, so image filtering is required in postprocessing. As with other interferometric optical techniques, the produced images are also wrapped to one wavelength of the light - the gradient of deformations of $(\partial W / \partial x)+n \lambda$, where $n$ is an arbitrary integer value, appears to have the same value. This results in the characteristic appearance of 'fringes'. Unwrapping algorithms [43] are used to remove the fringes, thus expand the maximum range of deformation the system can register. Unwrapping is the second step in the post-processing routine and is applied after the filtering.

Figure $2 \mathrm{~A}$ shows a $\Delta$-image of a vibrating beam. The $x$-direction represents the length of the beam, while the $y$-direction is the width. The image needs to be unwrapped before further processing. The shearography imaging sensor has a resolution of $1392 \times 1040$ pixels, but since a beam cannot fill the whole field of view of the sensor, a practical useful resolution will be lower. For a typical measurement, shown in Figure $2 \mathrm{~A}$, the measurement image is a matrix of approximate size $1150 \times 125$ (columns $\times$ rows). Each row can independently 


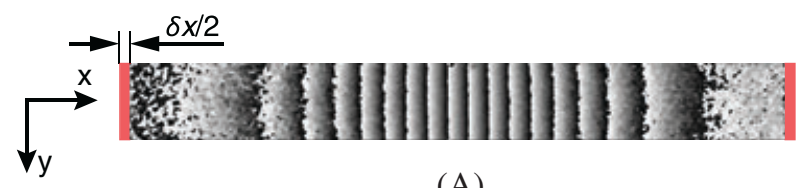

(A)

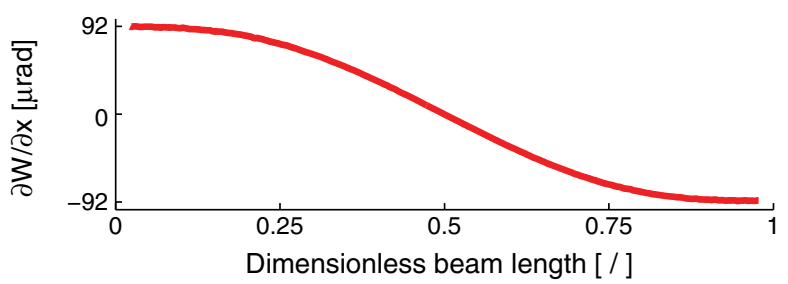

(B)

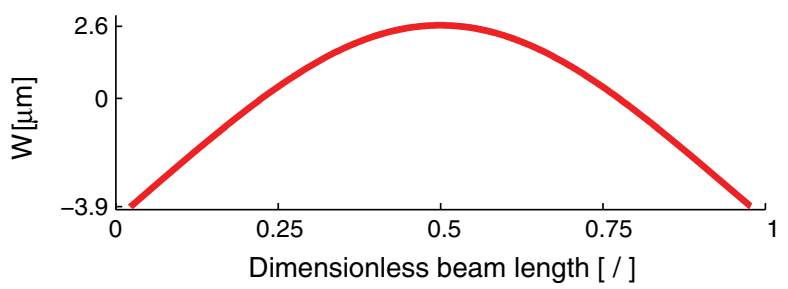

(C)

Figure 2: The $\Delta$-image of the vibrating beam (A) is filtered and unwrapped. Using Equation (1), the gradient of deformation (B) is calculated. The modal shape $(\mathrm{C})$ is acquired by integrating the deformation gradient

describe the gradient of deformation if Euler-Bernoulli beam theory is assumed, meaning that the 125 rows can be seen as sets of redundant data. This redundancy is used to eliminate noise, by averaging the beam data width-wise (averaging the rows). The image shown in Figure $2 \mathrm{~A}$ is first unwrapped and then averaged width-wise. The result after averaging widthwise can be seen in Figure 2B. Notice the lack of data at the left and right edges of the beam equivalent to $\delta x / 2$. By assuming that the beam is infinitely stiff in these small sections at both ends, the missing slopes can be taken equal to the first and last measured slope values respectively. Modal shape (Figure 2C) can be easily integrated from the gradient provided the integration constant is known. This integration constant is calculated during the stiffness identification, described in the subsequent section.

The images captured by the shearography have a speckled appearance due to the interference pattern created by the coherent light reflected from the object with a certain surface roughness. This 'speckle pattern' is required for the operation of shearography as the displacement is calculated on pixelby-pixel basis from it. In case the in-plane displacement is large, the speckle pattern will shift within the pixel, and the calculation of the $\Delta$-image will not be possible. In-plane displacement is the main reason for the speckle pattern decorrelation, but large out-of-plane displacement can also cause problems as it can distort the speckle pattern. Excessive shifting or distorting of the speckle pattern will result in the areas of the $\Delta$-images to appear as random noise, making the images unusable for further computations. The speckle decorrelation is the main limiting factor for the maximum deformation shearography can measure.

\section{Scanning laser vibrometry}

Like shearography, SLV allows for non-contact vibration measurements of a moving surface. A laser beam is a non-contacting transducer and avoids the problem of mass loading of the test specimen with hardware sensors. The vibration amplitude and frequency are extracted from the Doppler shift of the laser beam frequency due to the motion of the surface. The output of the SLV is a continuous analogue voltage that is directly proportional to the target velocity component along the direction of the laser beam.

A vibrometer is a two-beam laser interferometer that measures the frequency (or phase) difference between an internal reference beam and a test beam. The test beam is directed to the target, and scattered light from the target is collected and interfered with the reference beam on a CCD chip. The SLV adds a set of X-Y scanning mirrors, allowing the single laser beam to be moved across the surface of interest.

A Polytec scanning laser vibrometer is used to measure the response of the beam to the excitation signal. The Polytec system is a full-field system for automated vibration measurement, mapping, visualisation and analysis. It measures the modal amplitudes point by point in a scanning mode. The Polytec Scanning LVM software version 8.7.3.0 is used for analysing the signals used in this paper.

\section{Local stiffness identification}

The equation of motion of a continuous beam for a free vibration is $[44,45]$

$$
\frac{\partial^{2}}{\partial x^{2}}\left[\operatorname{EI}(x) \frac{\partial^{2} w(x)}{\partial x^{2}}\right]+\rho A(x) \frac{\partial^{2} w(x, t)}{\partial t^{2}}=0,
$$

where $A$ is the cross section area, $\rho$ is the specific mass, $E$ is the Young's modulus, $I$ is the section inertia, $x$ is the independent spatial coordinate and $t$ is the time. The solution can be written as a product of a time-dependent function $T(t)$ and a spatial-dependent function $W(x)$ :

$w(x, t)=W(x) T(t)$

Consider again a free-free beam vibrating in resonance at a circular frequency $\omega$. The time-independent differential equation describing the geometry of the modal shape $W$ $(x)$, assuming a constant specific mass, is 
$\frac{\mathrm{d}^{2}}{\mathrm{~d} x^{2}}\left[\operatorname{EI}(x) \frac{\mathrm{d}^{2} W(x)}{\mathrm{d} x^{2}}\right]-\rho A(x) \omega^{2} W(x)=0$

This equation can be seen as the differential equation of a beam loaded statically with a distributed pressure $p(x)=\rho A \omega^{2} W(x)$. Hence, the equation can be rewritten as

$\frac{\mathrm{d}^{2}}{\mathrm{~d} x^{2}}\left[\operatorname{EI}(x) \frac{\mathrm{d}^{2} W(x)}{\mathrm{d} x^{2}}\right]=p(x)$

$p(x)=\rho A \omega^{2} W(x)$.

Integration of the pressure yields

$\frac{\mathrm{d}}{\mathrm{d} x}\left[\operatorname{EI}(x) \frac{\mathrm{d}^{2} W(x)}{\mathrm{d} x^{2}}\right]=Q(x)$

$Q(x)=\int_{0}^{x} p(\xi) \mathrm{d} \xi$

$Q(x)$ is the transverse shear force at position $x$. In case of a free-free beam with length $L$, the shear force is zero at the beam boundaries $x=0$ and $x=L$.

Integration of the transverse shear force gives

$\operatorname{EI}(x) \frac{\mathrm{d}^{2} W(x)}{\mathrm{d} x^{2}}=M(x)$

$M(x)=\int_{0}^{x} Q(\xi) \mathrm{d} \xi$

The identification of the local beam stiffness EI $(x)$ uses the relation between the bending moment $M$ and the curvature in the considered point [12]:

$\operatorname{EI}\left(x_{i}\right) \frac{\mathrm{d}^{2} W\left(x_{i}\right)}{\mathrm{d} x^{2}}=M\left(x_{i}\right)$

$\operatorname{EI}\left(x_{i}\right)=\frac{M\left(x_{i}\right)}{\frac{\mathrm{d}^{2} W\left(x_{i}\right)}{\mathrm{d} x^{2}}}$

The evaluation of $E I$ in a single point $x_{i}$ requires the knowledge of $M\left(x_{i}\right)$ and the second derivative of the modal shape $W$ (in the case of SLV) or the derivative of the modal shape slope (in the case of shearography).

Once the bending moment $M(x)$ is derived, the beam stiffness EI of the beam can be evaluated point by point by means of Equation (12).

Figure 3 shows the mutual relations between the pressure $p$, the transverse shear force $Q$ and the bending moment $M$. In the case of SLV, all are derived from the modal shape $W$, while in case of shearography, they are consecutively integrated from the slope of the modal shape $\partial W / \partial x$.

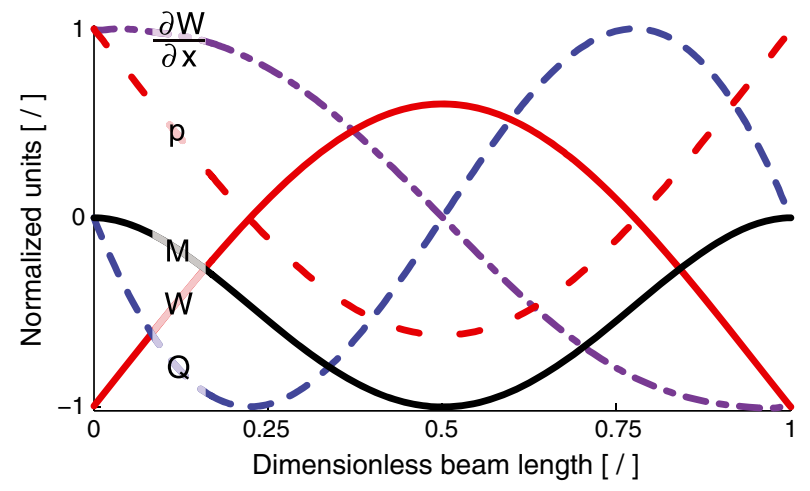

Figure 3: The modal shape $W$ and the slope of the modal shape $\partial W / \partial x$, along with pressure $p$, transverse shear force $Q$ and bending moment $M$ of a flat beam, in normalised units

Experimental data usually contain a vast amount of noise. Due to the presence of this noise, the measured raw data cannot be used directly. In this research, the raw data were smoothed by fitting the experimental data with a superposition of high-degree Lagrange shape functions. Lagrange shape functions are polynomials capable of producing highly accurate fits with a small number of nodal points. The fitting of the measured points is based on a least squares procedure and establishes the displacement values in the nodal points. If the displacement values (in the case of SLV) or the slope values (in the case of shearography) in the nodal points are known, the value of a point in an arbitrary position can be interpolated.

The measured values are fitted by a superposition of $M$ Lagrange polynomials $L_{j} . M$ is the number of selected nodal points:

$V(x)=\sum_{j=1}^{M} L_{j} V_{j}$

$V(x)$ can represent the displacement as well as the slope:

$L_{j}=\prod_{1 \leq k \leq M} \frac{x-x_{k}}{x_{j}-x_{k}}$

$k \neq j$

As can be verified in the above expression, the Lagrange polynomials $L_{j}$ take the value 1 for $x=x_{j}$ and the value 0 in all other nodal points $x_{j}$.

The principle of dividing the beam into a regular grid of nodal points for the Lagrange functions, including the end points, guarantees stability of the curve fit over the whole beam. The number of selected nodal points $M$ defines the polynomial degree of the curve fitting function. A more complex modal shape requires more nodal points. The complexity of the modal shape is a function of the variation of the local beam stiffness distribution. The lowest 
complexity case is a beam with an overall constant stiffness. The interpolation capacity of Lagrange functions was investigated in [46] where it was shown that the modal shape of homogeneous beams and plates can be exactly curve fitted with Lagrange functions with a polynomial degree of 7. More variations of the local stiffness will require a higher polynomial degree. Since the variation of the local stiffness (hence curvature) is not a priori known, the best way to establish a suitable value for $M$ is to increase systematically the number until the computed value of the signal-to-noise ratio (SNR) is not changing any more by the further increase of $M$. The number of experimental points $N$ must be much higher than the number of nodal points $M$, to avoid the influence of measurement noise on the curve fitting. A factor 10 in the $N / M$ ratio guarantees that the modal shape is properly fitted while avoiding the influence of measurement noise. In this paper, the value of $M=19$ is used for all curve fitting purposes, along with an $N / M$ ratio of 20 or more.

The coefficients $V_{j}$ in the expression (13) are found by curve fitting of the measured values. $V_{j}$ can be found by the minimisation of the least square error $\varepsilon$ between the fitted and measured values $V_{i}^{e}$, summed over the $N$ measurement points:

$\varepsilon=\sum_{i=1}^{N}\left[\sum_{j=1}^{M} L_{j}\left(x_{i}\right) V_{j}-V_{i}^{e}\right]^{2}$

Minimisation of $\varepsilon$ with respect to the parameters $V_{k}$ gives

$\frac{\partial \varepsilon}{\partial V_{k}}=\sum_{i=1}^{N} 2\left[\sum_{j=1}^{M} L_{j}\left(x_{i}\right) V_{j}-V_{i}^{e}\right] L_{k}\left(x_{i}\right)=0$,

which leads to a set of $M$ linear equations in $V_{j}$ :

$A_{j k} V_{j}=b_{k}$,

where

$$
\begin{aligned}
A_{j k} & =\sum_{j=1}^{M} \sum_{i=1}^{N} L_{j}\left(x_{i}\right) L_{k}\left(x_{i}\right), \\
b_{k} & =\sum_{i=1}^{N} V_{i}^{e} L_{k}\left(x_{i}\right) .
\end{aligned}
$$

The free-free boundary conditions of the test beam require a translational and rotational equilibrium. If this is not the case, the shear force $Q$ and the bending moment $M$ computed with Equations (8) and (10) will not be zero at $x=L$.

Deviations from these equilibrium conditions can occur due to experimental errors, the curve fit procedure and the integration procedure.
In order to impose translational and rotational equilibrium, the curve fitted results are superposed by two rigid body modes - a pure translation and a pure rotation in $x=L / 2$ :

$$
\begin{aligned}
W(x)_{\text {corrected }}=W(x)_{\text {fitted }} & +a W(x)_{\text {translation }} \\
& +b W(x)_{\text {rotation }}
\end{aligned}
$$

with

$$
\begin{aligned}
W(x)_{\text {translation }} & =1, \\
W(x)_{\text {rotation }} & =\frac{2 x}{L}-1 .
\end{aligned}
$$

The requirements are as follows:

$$
\begin{aligned}
& Q(x=L)=\rho A \omega^{2} \int_{0}^{L} W_{\text {corrected }}(x) \mathrm{d} x=0 \\
& M(x=L)=\rho A w^{2} \int_{0}^{L} x W_{\text {corrected }}(x) \mathrm{d} x=0
\end{aligned}
$$

The parameters $a$ and $b$ in Equation (18) must be determined in such a way that the requirements (19) and (20) are fulfilled. This leads to the following:

$a=-\frac{Q(x=L)}{\omega^{2} \rho A L}$

$b=-\frac{6 M(x=L)}{\omega^{2} \rho A L^{2}}-3 a$

In the above expression, $Q$ and $M$ are computed with the uncorrected smoothed and curve fitted data.

\section{Measurement Set-ups}

The test specimens are aluminium beams in three different geometries - the flat, convex and concave beam. The flat beam has a constant thickness of $4 \mathrm{~mm}$, the convex has a thickness varying from $2 \mathrm{~mm}$ at the edges to $4 \mathrm{~mm}$ in the centre and the concave beam's thickness is $4 \mathrm{~mm}$ at the edges and $2 \mathrm{~mm}$ in the centre. The Young's modulus of the aluminium is measured to be $E=68.8 \mathrm{GPa}$, using the resonalyser method [47]. The exact geometries of the beams are measured precisely. The thickness distribution is used to calculate the distribution of the local flexural rigidity, EI, which in turn is used for comparison to the identified results. For example, the flat beam with thickness $T=4 \mathrm{~mm}$ has a constant flexural rigidity of $E I=8.9 \mathrm{Nm}^{2}$. Other properties are summarised in Table 1.

To properly image the beam vibrations with shearography, the surface needs to be diffusely reflective. The aluminium beams have a shiny metallic surface, so a thin coating of white 
Table I: Properties of the aluminium test beams used for tests

\begin{tabular}{lcccc}
\hline Beam & Length & Width & Mass & Resonant frequency \\
\hline Flat & $L[\mathrm{~mm}]$ & $B[\mathrm{~mm}]$ & $\mathrm{m}[\mathrm{g}]$ & $\mathrm{fl}_{\mathrm{i}}[\mathrm{Hz}]$ \\
Convex & 220.5 & 25.0 & 58.3 & 427.5 \\
Concave & 220.1 & 25.0 & 49.1 & 453.6 \\
\hline
\end{tabular}

powder is sprayed over the surface in order to be able to image it properly. The coating is very thin $(20-30 \mu \mathrm{m})$, so it does not influence the stiffness or the mass of the beam. The SLV does not need this kind of surface treatment.

To approach the required free-free boundary condition, the beam is suspended on a rigid frame by very soft elastic cords. Additionally, the beam rests against two small pins wrapped with soft material to prevent rigid body oscillations during the measurement. The cords and the pins have little influence on the natural frequency or the modal shape, even if they are slightly misaligned to the nodal points. Their purpose is to limit rigid body oscillations which can cause local spikes in the modal shape in case of SLV measurements and excessive noise in case of shearography. For the sake of precise measurements, however, the nodal points are located as accurately as possible. In case of a beam with unknown stiffness distribution, the position of cords and pins can be iteratively improved before the actual measurements by using high excitation levels (millimetre instead of micrometres). For such high amplitudes, a non-correct position causes the beam to bounce off the pins, which can be visually observed. Figure 4 shows a suspended test beam on the set-up.

To isolate the outside vibrations, the shearography camera, along with the frame holding the beam, is placed on an active vibration isolating table. The table is kept level and vibration free using pneumatically actuated legs.

The beam is excited acoustically with a sinusoidal signal using a small loudspeaker (Figure 4A). An acoustical excitation is used in order to avoid physical contact and thus avoid mass loading of the test beam. In this paper, it is shown that the identification can be successful by only using the first resonant frequency. The associated first modal shape is also the most easy to excite acoustically. The vibration amplitude can be controlled by the volume of the loudspeaker.

The data acquired from shearography come in the form of a $\Delta$-image, representing the gradient of deformation $\partial W / \partial x$. For a typical measurement presented in this paper, the measurement image is a matrix of approximate size $1150 \times 125$. When the data are averaging width-wise, the resulting $\partial W / \partial x$ is captured at approximately 1150 points. The number of points the SLV set-up can acquire is limited by the angular resolution of the scanner head. For our setup, the scanning was performed at maximum density - 360 points per length of the beam. The shearography can thus acquire information on the modal shape of the vibrating beam in three times higher density than SLV.

The acquisition time for both techniques can vary, but typically for shearography, a $\Delta$-image is acquired in 3-4 s. An SLV scan of 360 points lasts around $6 \mathrm{~min}$.

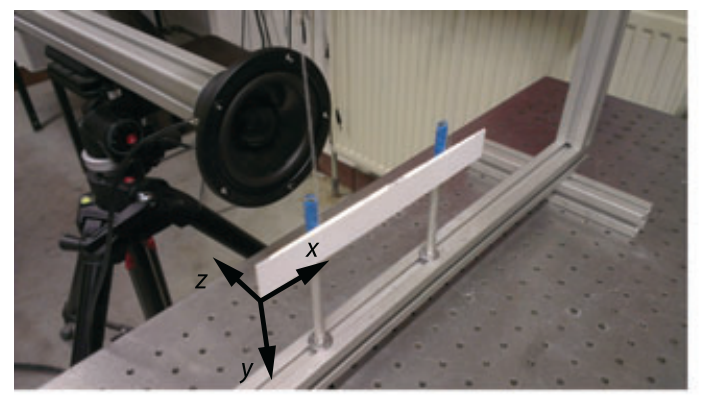

(A)

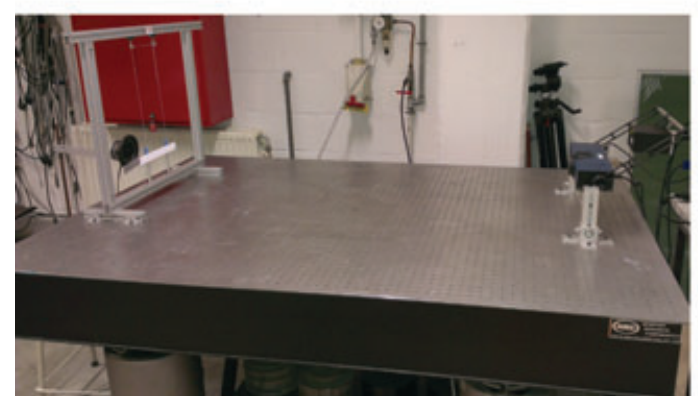

(B)

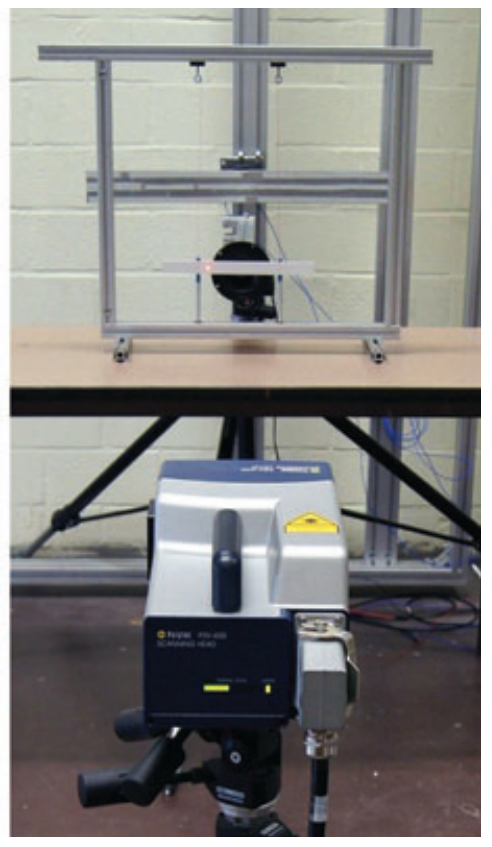

(C)

Figure 4: Measurement set-ups for shearography (B) and the SLV (C), with a close-up on the vibrating beam (A) 


\section{Estimating the Noise}

The amount of noise in the acquired modal shape information for both shearography and the SLV is quantified using the SNR. The definition used to compute the SNR is [48]

$\mathrm{SNR}=\frac{\mu(S)}{\sigma(N)}$,

where the $\mu(S)$ is the mean value of the signal $S$ and $\sigma(N)$ is the standard deviation of the estimate of the noise $N$. Signal $S$ is the scanned modal shape, $W$, in case of the SLV, or the acquired gradient of the modal shape, $\partial W / \partial x$, in case of shearography. Furthermore, only signals in the range $(0.25,0.75)$ of the beam length $L$ are used as only in this range of the beam length the stiffness will actually be identified. The curvature outside this range is nearly zero and hence cannot be used for accurate identification of the stiffness. The noise $N$ is estimated as the difference between the signal and its curve fit,

$N(x)=W(x)-W_{\text {fit }}(x) \quad$ for SLV,

$N(x)=\frac{\partial W}{\partial x}(x)-\left(\frac{\partial W}{\partial x}\right)_{\text {fit }}(x) \quad$ for shearography.

SNR needs to be defined as a positive value. The gradient of the modal shape, $\partial W / \partial x$, and the modal shape $W$ can have both positive and negative values, so the absolute value of the ratio of the mean $\mu(S)$ to $\sigma\left(S-S_{\text {fit }}\right.$ is used:

$\mathrm{SNR}=\left|\frac{\mu(S)}{\sigma\left(S-S_{\text {fit }}\right)}\right|$

In Figure $5 \mathrm{~A}, \mathrm{~B}$, the most important parameters when calculating the SNR for both SLV and shearography measurements are illustrated.

For SLV, a series of measurements were made by varying the volume of the acoustic excitation of the beams. The measured mean amplitudes, $\mu|W|$, were recorded from 3 to $0.5 \mathrm{~mm}$, proving the ability of the SLV to acquire a broad range of vibrations. For the lowest amplitude levels, the SNR is, predictably, low. At mean amplitudes, $\mu|W|>1 \mu \mathrm{m}$ values of SNR $>1000$ are recorded. As the volume of the acoustic excitation is increased further, the amplitudes increase up to $0.5 \mathrm{~mm}$. However, this increase in amplitude does not improve the SNR. For the flat beam, the SNR actually decreases significantly at amplitudes above $10 \mu \mathrm{m}$. Possible reasons include the loss of focus of the laser and vibrational interaction with the frame the beam is attached to. As the vibrations approach the millimetre amplitude level, the non-linear effects become more pronounced so the displacements cannot be assumed to be limited to

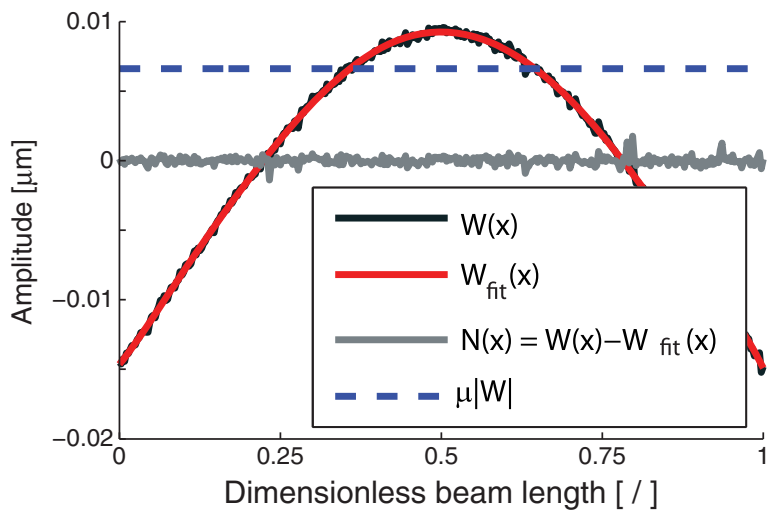

(A)

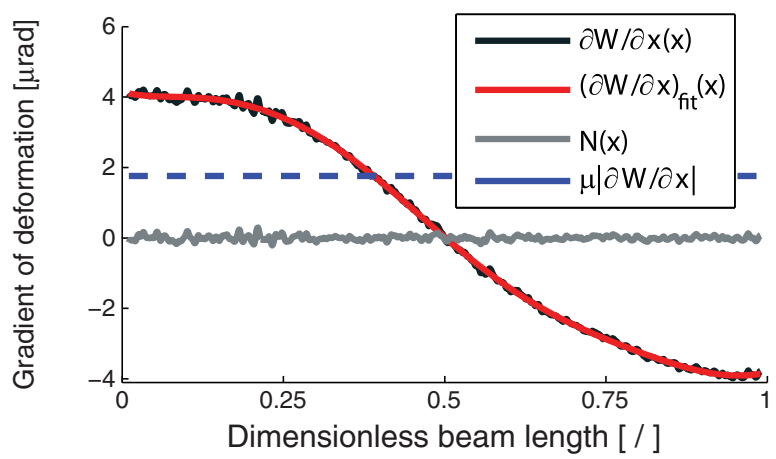

(B)

Figure 5: Signal, $S$, is curve fitted to calculate the noise, $N$. The examples with low SNR are given for $\mathrm{SLV}, \mathrm{SNR}=27$, (A) and shearography, $\mathrm{SNR}=31$, (B)

out-of-plane. The excitation for the measurements with SLV was increased until the sound volume in the laboratory became uncomfortably high. From Figure 6 , it can be concluded that the vibration amplitudes in the range from 1 to $10 \mu \mathrm{m}$ give the best results. For that level of excitation, the SNR is consistently high and the sound volume is at the limit of audible sound.

The shearography measurements depend on the shearing distance that is introduced by the measurement set-up. The higher the shearing distance, the higher the sensitivity of the method. But, high shearing distances carry the problem of large edge areas that cannot be resolved, and the unwrapping algorithm might have problems since the high sensitivity results in more fringes. For this reasons, different shearing distances were used to select the one which works best for our problem case. The shearing can be controlled by the angle of the shearing mirror but is measured on the specimen itself as the distance between the two sheared images. Shearing was selected to be 10,20 and $40 \%$ of the maximum shearing angle, $\alpha_{\max } \approx 0.5^{\circ}$. This corresponds to $\delta x=\{2.4,4.9,9.8\} \mathrm{mm}$, as measured on the object at $1.4 \mathrm{~m}$ distance. Higher shearing distances were not used as this would increase the unresolved edge area to unacceptable values for stiffness identification. 


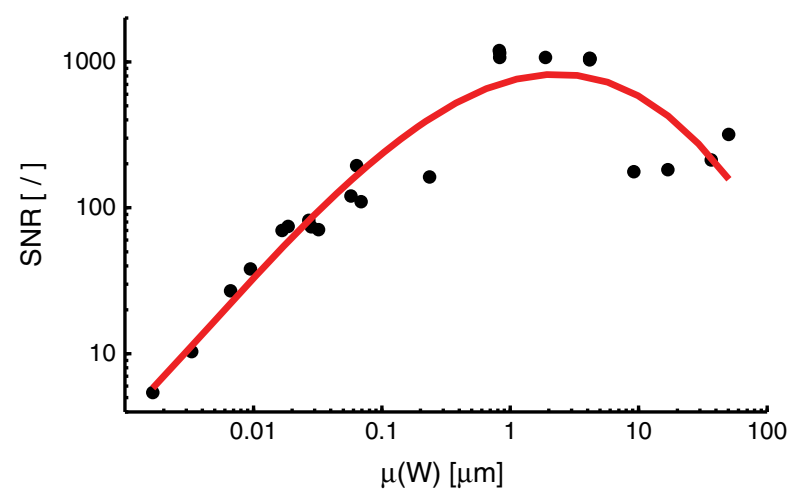

(A)

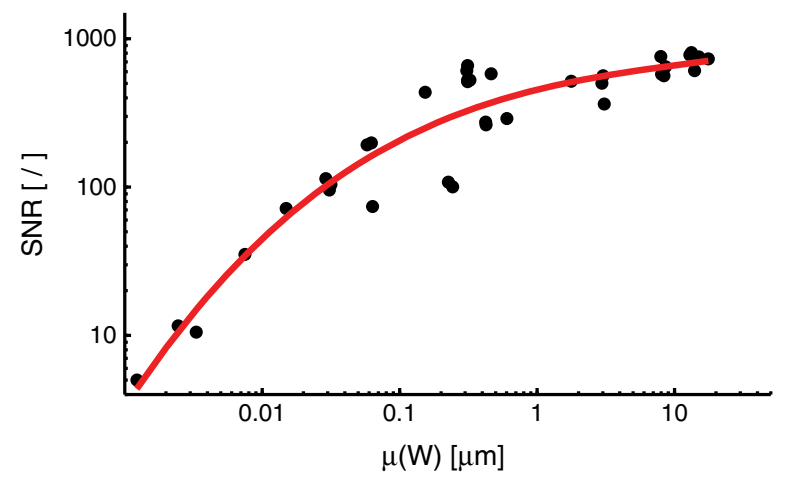

(B)

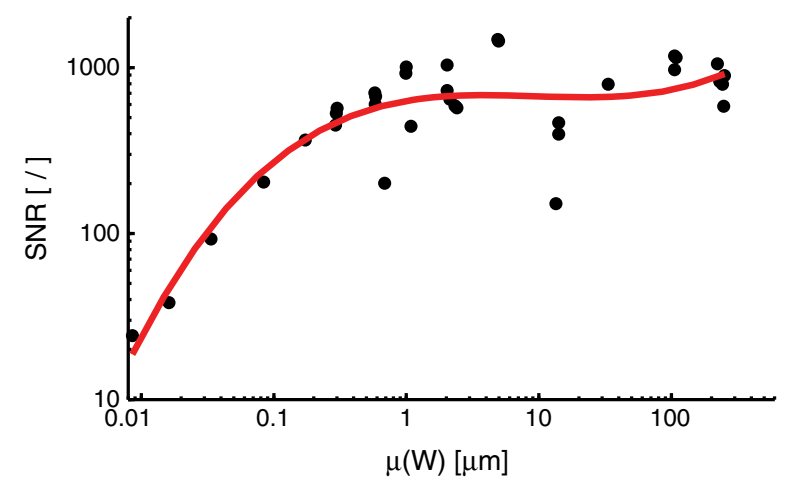

(C)

Figure 6: SNR for SLV measurements of the flat (A), convex (B) and concave $(\mathrm{C})$ beams

Figure 7A shows the influence different shearing distance values have on the SNR. It can be expected that higher shearing distance value would have higher SNR since the signal will be increased (higher sensitivity) and the noise would remain constant. The highest shearing distance $(\delta x=9.8 \mathrm{~mm})$ did indeed yield the highest SNR values, so this value was used in subsequent measurements, seen in Figure 7B,C.

In Figure 7, an increase of the SNR as the amplitude of excitation can be observed. At a certain point, however, the amplitude that the beam is vibrating at (particularly the ends of the beam) starts causing the speckle decorrelation. Speckle decorrelation is a phenomenon

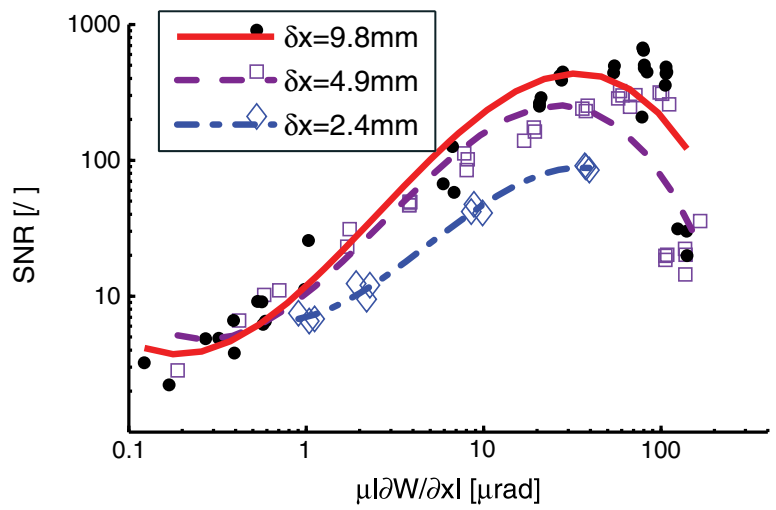

(A)

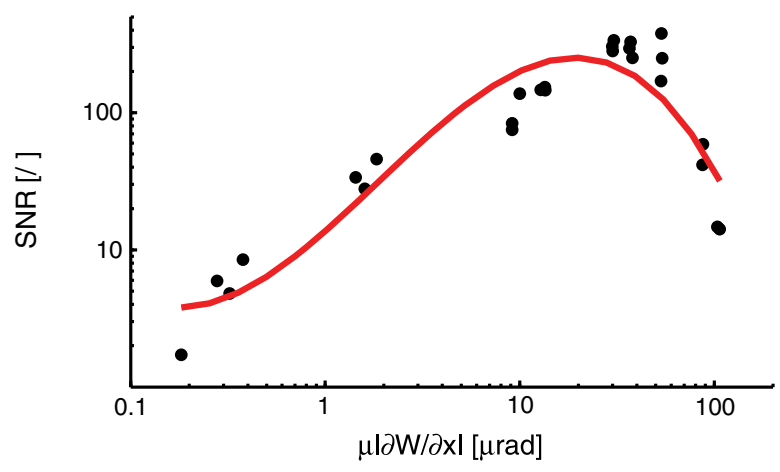

(B)

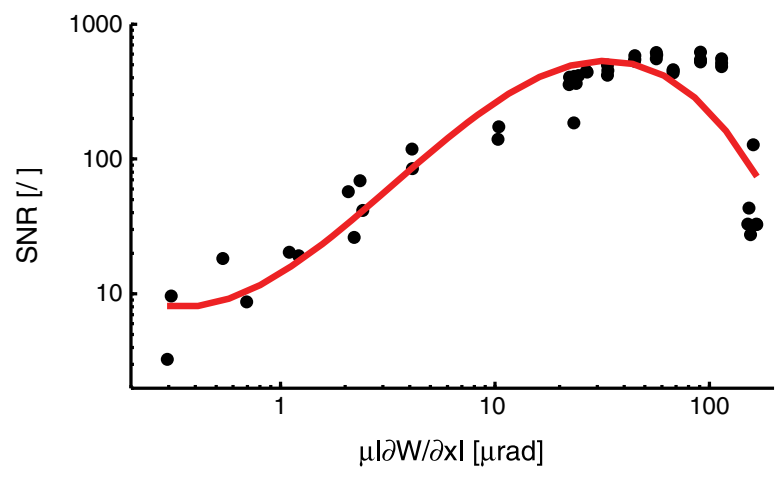

(C)

Figure 7: SNR for shearography measurements of the flat (A), convex (B) and concave (C) beams. The flat beam is measured using several shearing distances. The highest shearing distance, $\delta x=9.8$ $\mathrm{mm}$, was chosen for measurements of convex and concave beams

where large out-of-plane or in-plane deformations cause speckles to move as observed by the sensor of shearography. In that case, the speckles cannot be directly compared, and the shearography algorithm fails. The areas of the beam which crosses the threshold for speckle decorrelation appear as pure noise, so even the mean value $\mu|\partial W / \partial x|$ does not give reliable results. As the excitation is increased past the threshold where speckle decorrelation appears, the registered value of $\mu|\partial W / \partial x|$ appears to stay unchanged. This can be seen as a sudden drop in SNR at $\mu|\partial W / \partial x| \sim 150 \mu \mathrm{rad}$. 
Table 2: Comparison of highest achieved SNR for both methods

\begin{tabular}{lcc}
\hline & Shearography & SLV \\
\cline { 2 - 3 } Beam & maximal SNR [/] & maximal SNR [/] \\
\hline Flat & 675 & 1191 \\
Convex & 434 & 811 \\
Concave & 619 & 1477 \\
\hline
\end{tabular}

The best results acquired by shearography in our experiments (there is some variability depending on the beam used for measurements) are those in the range $(50,100) \mu \mathrm{rad}$. The modal shape slope $\partial W / \partial x$ can be integrated to obtain the modal shape, so that the modal shape amplitude can be directly compared to the one of SLV. The range of $\mu|\partial W / \partial x|=(50,100) \mu \mathrm{rad}$ corresponds to the range of $\mu|W|=(7,15) \mu \mathrm{m}$. The range of shearography is clearly more limited than the one of SLV. Since the acoustic excitation volume can be freely adjusted to put the vibrations of the beam in the optimal acquisition range, this limitation of shearography is not significant for our application.

Still, results of shearography have a lower SNR than the measurements under the same conditions, on the same specimens using SLV. As seen in Table 2, the SLV can obtain 1.7-2.4 times higher SNR values. Shearography also has a problem at the edges of the beam where the gradient $\partial W / \partial x$ cannot be measured. These differences will be further discussed in the following section where the results for the stiffness identification are compared.

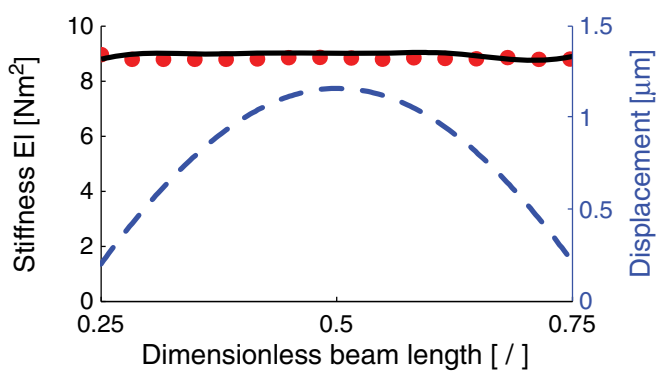

(A) Flat beam, SLV

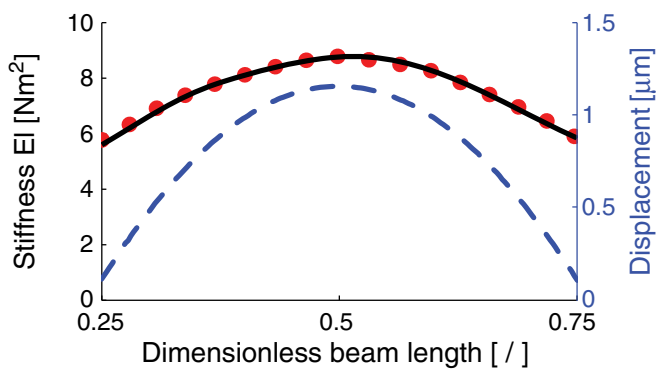

(C) Convex beam, SLV

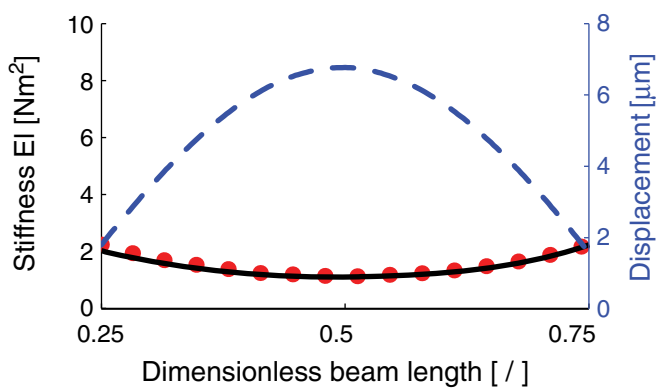

(E) Concave beam, SLV

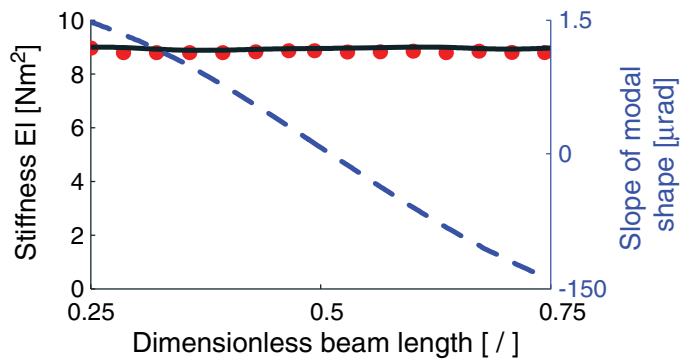

(B) Flat beam, shearography

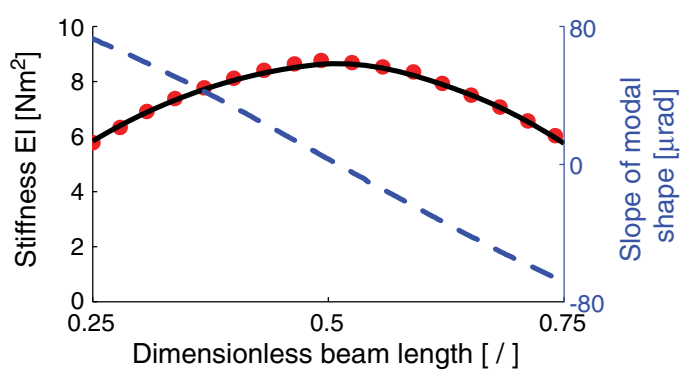

(D) Convex beam, shearography

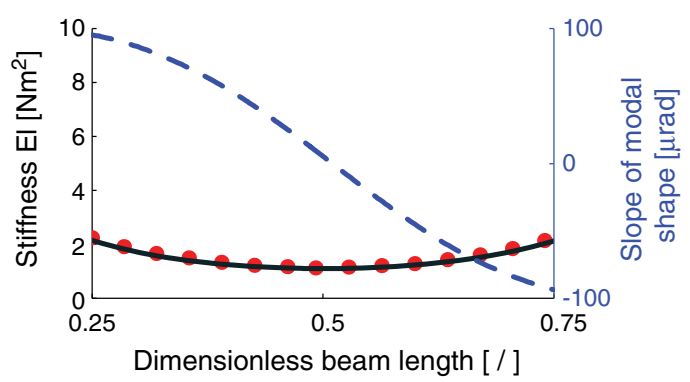

(F) Concave beam, shearography

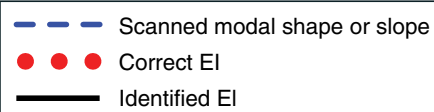

Figure 8: Best results of stiffness identifications from SLV and shearography for the three types of beams 


\section{Identification Error}

The correct $E I$ is calculated from $E$, measured by the resonalyser test [47], and from the moment of inertia $I$ of a section as

$$
\begin{aligned}
\operatorname{EI}_{\text {correct }}\left(x_{i}\right) & =E_{\text {resonalyser }} I\left(x_{i}\right) \\
= & E_{\text {resonalyser }} \frac{B\left(x_{i}\right) T\left(x_{i}\right)^{3}}{12}
\end{aligned}
$$

where $B$ is the measured local width and $T$ the measured local thickness of the beam.

The error of the identification is calculated as the mean value of the absolute error ratio in the range $0.25-0.75$ of the beam length:

$\varepsilon_{\mathrm{EI}}=\frac{1}{N} \sum_{i=1}^{N} \frac{\left|\mathrm{EI}_{\text {identified }}\left(x_{i}\right)-\mathrm{EI}_{\text {correct }}\left(x_{i}\right)\right|}{\mathrm{EI}_{\text {correct }}\left(x_{i}\right)}$

In Figure 8, the results for the most successful identifications using data from both SLV and shearography are presented. Qualitatively, it can be seen that the stiffness EI is identified very accurately. Errors of identification $\varepsilon_{\mathrm{EI}}$ are quantified in Table 3 . The flat and convex beams show the mean identification error $\varepsilon_{\mathrm{EI}}$ between 1 and $2 \%$. Concave beam shows comparably a high error $\varepsilon_{\mathrm{EI}}$ for both SLV and shearography. The concave beam is the most flexible and as such the most fragile of the three beams, and some micro-cracks in the surface can occur during manufacturing. Effects of imperfections in the beam are not taken into account in Equation (27), so some deviations between real stiffness and the $E I_{\text {correct }}$ can occur. These deviations would increase the apparent identification error.

SLV consistently produces higher SNR (approximately by a factor of 2) for a given specimen and amplitude of vibrations. However, the information per length of the beam produced by shearography is three times denser, compared to the SLV. The computation of the curvature with only one step differentiation also improves the values of the stiffness distribution obtained by shearography. Despite consistently having lower SNR values, identification

Table 3: Comparison of best achieved $\boldsymbol{\varepsilon}_{\mathbf{E I}}$ along with their SNR for both methods

\begin{tabular}{llcc}
\hline Beam & Method & SNR [/] & $\varepsilon_{\text {El }}[\%]$ \\
\hline Flat & Shearography & 645 & 1.43 \\
& SLV & 1191 & 1.77 \\
Convex & Shearography & 434 & 0.82 \\
& SLV & 757 & 0.83 \\
Concave & Shearography & 615 & 4.71 \\
& SLV & 1445 & 7.43 \\
\hline
\end{tabular}

from slopes using shearography produces lower errors $\varepsilon_{\mathrm{EI}}$ for all the beams, compared to SLV. The good accuracy obtained from results of shearography also indicates that the lack of information at the ends of the beam, of width $\delta x / 2$ at each end, does not pose a hindrance.

For a given specimen, using either shearography or SLV, the SNR of the measurement is a very good indicator and predictor whether the stiffness identification will be successful. Measurements with low SNR values are giving bad identification results. The results with the best $\varepsilon_{\mathrm{EI}}$ are the ones with the highest SNR.

\section{Conclusion and Further Work}

Identification of the local stiffness distribution of beams is possible by dividing the bending moment by the curvature. The bending moment can be computed by double integration of the dynamic pressure caused by a modal shape vibration at the first natural frequency. Only the central 50\% of the beam shows enough curvature for stiffness identification. The modal shape can be obtained both by SLV and by shearography. A good operating range for the amplitude values for SLV and slope values for shearography has been established in this paper. The uncertainty of the obtained stiffness values was always lower than 7.5\% for SLV and lower than 5\% for shearography. SLV has the advantage of having a better SNR; it yields complete modal information, needs no vibration isolation and needs no surface treatment of the test beams. Disadvantages of SLV, as compared to shearography, are the lower density of measurement points, the much longer measurement time (6 min versus $4 \mathrm{~s}$ for shearography) and the necessity for a two-step differentiation of the modal amplitudes to obtain the local curvatures. Shearography's advantages are the lower equipment cost and the ability to quickly produce full-field results. Shearography's lower SNR is compensated for by a three times denser grid of measurement points. The data from shearography require only one derivation step to obtain the local values of the curvatures.

In this paper, a conclusion is established that shearography is a more suitable method for stiffness identification of beams than SLV. The advantage of the full-field measurements by shearography will be even more pronounced in case of 2D stiffness identification of plates. The scanning time for a $360 \times 360$ grid of points with the SLV would need $360 \times 6 \mathrm{~min}$, which is $36 \mathrm{~h}$. Our future research on plate stiffness distributions will hence be conducted with shearography, while SLV can be used as a supporting and validation technique.

The use of shearography for the local 2D stiffness distribution will provide additional difficulties as compared with 1D case of beams presented in this paper. A major difficulty to overcome is computing all six components of the plate stiffness for each point, in the case of the assumed anisotropy (which must be assumed in the case of damage). 
The possible number of measured curvatures is only 3 $\left(\partial^{2} W / \partial x^{2}, \partial^{2} W / \partial y^{2}\right.$ and $\left.\partial^{2} W / \partial x \partial y\right)$, so more modal shapes have to be included. We aim to measure torsion and two bending modal shapes of the test plate. Clustered measurement points with equal assumed stiffness can be taken into account if more data per calculation point are needed.

\section{ACKNOWLEDGEMENT}

Part of this research has been sponsored by FWO, the Fund for scientific research of Flanders, Belgium.

\section{REFERENCES}

1. Banan, M. and Hjelmstad, K. (1994) Parameter estimation of structures from static response. I: computational aspects. J. Struct. Eng. 120, 3243-3258.

2. Banan, M. and Hjelmstad, K. (1994) Parameter estimation of structures from static response. II: numerical simulation studies. J. Struct. Eng. 120, 3259-3283.

3. Rucka, M. and Wilde, K. (2006) Crack identification using wavelets on experimental static deflection profiles. Eng. Struct. 28, 279-288.

4. Buda, G. and Caddemi, S. (2008) Identification of concentrated damages in Euler-Bernoulli beams under static loads. j. Struct. Mech. 133, 942-956.

5. Yang, Z., Liu, G. and Lam, K. (2002) An inverse procedure for crack detection using integral strain measured by optical fibers. Smart mat. struct. 11, 72-78.

6. Li, L., Ghrib, F. and Polies, W. (2009) Identification of stiffness distribution and damage in Euler-Bernoulli beams using static response. in Proceedings of Cansmart 2009 International workshop on smart materials and structures, no. October, pp. 207-216.

7. Sztefek, P. and Olsson, R. (2007) Inverse method for stiffness determination of impact damage in composites. in Proceedings of the annual SEM conference.

8. Grediac, M., Pierront, F., Avrilt, S. and Toussaint, E. (2006) The virtual fields method for extracting constitutive parameters from full-field measurements a review. Strain 42 233-253.

9. Devivier, C., Pierron, F. and Wisnom, M. (2012) Damage detection in composite materials using deflectometry a fullfield slope measurement technique. Compos. Appl. Sci. Manuf. 43, 1650-1666.

10. Salawu, O. (1997) Detection of structural damage through changes in frequency a review. Eng. Struct. 19, 718-723.

11. Kim, J.-T., Ryu, Y.-S., Cho, H.-M. and Stubbs, N. (2003) Damage identification in beam-type structures: frequency-based method vs mode-shape-based method. Eng. Struct. 25, 57-67.

12. Maeck, J. and De Roeck, G. (1999) Dynamic bending and torsion stiffness derivation from modal curvatures and torsion rates. J. Sound Vib. 225, 153-170.

13. Ndambi, J.-M., Vantomme, J. and Harri, K. (2002) Damage assessment in reinforced concrete beams using eigenfrequencies and mode shape derivatives. Eng. Struct. 24, 501-515.

14. Kim, H. and Melhem, H. (2004) Damage detection of structures by wavelet analysis. Eng. Struct. 26, 347-362.
15. Liu, G. and Chen, S. (2002) A novel technique for inverse identification of distributed stiffness factor in structures. J. Sound Vib. 254, 823-835.

16. Kokot, S. and Zembaty, Z. (2009) Vibration based stiffness reconstruction of beams and frames by observing their rotations under harmonic excitations-numerical analysis. Eng. Struct. 31, 1581-1588.

17. Friswell, M. I. (2007) Damage identification using inverse methods. Phil. Trans. A Math. Phys. Eng. Sci. 365, 393-410.

18. Doebling, S., Farrar, C., Prime, M. and Shevitz, D. (1996) Damage identification and health monitoring of structural and mechanical systems from changes in their vibration characteristics: a literature review. Tech. Rep. Pt 10, Los Alamos National Lab., NM (United States).

19. Farrar, C. and Doebling, S. (1997) An overview of modal-based damage identification methods. Proceedings of DAMAS Conference, Sheffield.

20. Farrar, C., Doebling, S. and Duffey, T. (1999) Vibration-based damage detection. in SD2000 Structural Dynamics Forum.

21. Farrar, C., Doebling, S. and Nix, D. A. (2001) Vibration-based structural damage identification. Phil. Trans. A Math. Phys. Eng. Sci. 359, 131-149.

22. Steinchen, W. and Yang, L. (2003) Digital Shearography: Theory and Application of Digital Speckle Pattern Shearing Interferometry. SPIE Press, Bellingham, Washington, USA.

23. Francis, D., Tatam, R. P. and Groves, R. M. (2010) Shearography technology and applications: a review. Meas. Sci. Tech. 21, $1-29$.

24. Leendertz, J. A. and Butters, J. N. (1973) An image-shearing speckle-pattern interferometer for measuring bending moments. J. Phys. E Sci. Instrum. 6, 1107-1110.

25. Hung, Y. Y.. (1982) Shearography: a new optical method for strain measurement and nondestructive testing. Opt. Eng. 21, 391-395.

26. Hung, Y., Chen, Y., Ng, S., Liu, L., Huang, Y., Luk, B., Ip, R., Wu, C. and Chung, P. (2009) Review and comparison of shearography and active thermography for nondestructive evaluation. Mater. Sci. Eng. R Rep. 64, 73-112.

27. Staszewski, W., Boller, C. and Tomlinson, G. (2004) Health monitoring of aerospace structures.

28. Hung, Y. (1999) Applications of digital shearography for testing of composite structures. Compos. B Eng. 30, 765-773.

29. Mäckel, P., Heyen, H. and Steinchen, W. (2007) Die Scherografie: Ein quantitatives Messverfahren zur Schwingungs- messung und zerstörungsfreien Prüfung. Laser Technik Journal 2, 49-54.

30. Hung, Y., Luo, W., Lin, L. and Shang, H. (2000) Evaluating the soundness of bonding using shearography. Compos. Struct. 50, 353-362.

31. Lai, W. L., Kou, S. C., Poon, C. S., Tsang, W. F., Ng, S. P. and Hung, Y. Y. (2009) Characterization of flaws embedded in externally bonded CFRP on concrete beams by infrared thermography and shearography. J. Nondestr. Eval. 28(1), 27-35.

32. Hung, Y. and Ho, H. (2005) Shearography: an optical measurement technique and applications. Mat. Sci. Eng.: R: Reports 49, 61-87. 
33. De Angelis, G., Meo, M., Almond, D., Pickering, S. and Angioni, S. (2012) A new technique to detect defect size and depth in composite structures using digital shearography and unconstrained optimization. NDT \& E Intern. 45, 91-96.

34. Yang, L., Steinchen, W., Kupfer, G. and Vo, F. (1998) Vibration analysis by means of digital shearography. Opt. Lasers Eng. 30, 199-212.

35. Goto, D. T. and Groves, R. M. (2010) Error analysis of 3D shearography using finite-element modelling. in Proceedings of SPIE 7718, 771816-1 - 771816-12.

36. Goto, D. T. and Groves, R. M. (2010) A combined experiment with simulation approach to calibrated 3D strain measurement using shearography. in Proceedings of SPIE 7387, 73871J73871J-10.

37. Giraudeau, A. and Pierron, F. (2005) Identification of stiffness and damping properties of thin isotropic vibrating plates using the virtual fields method: theory and simulations. J. Sound Vib. 284, 757-781.

38. Kim, J.-H., Pierron, F., Grediac, M. and Wisnom, M. R. (2007) A procedure for producing reflective coatings on plates to be used for full-field slope measurements by a deflectometry technique. Strain 43, 138-144.

39. Hild, F. and Roux, S. (2006) Digital image correlation: from displacement measurement to identification of elastic properties - a review. Strain 42, 69-80.
40. Jacquot, P. (2008) Speckle interferometry: a review of the principal methods in use for experimental mechanics applications. Strain $\mathbf{4 4}$, 57-69.

41. Sutton, M. A., Orteu, J.-J. and Schreier, H. (2009) Image Correlation for Shape, Motion and Deformation Measurements: Basic Concepts, Theory and Applications. Springer Publishing Company, Incorporated, New York, USA.

42. Mäckel, P. (2010) User manual and service instructions for SE3 Shearography/ESPI.

43. Ghiglia, D. C. and Pritt, M. D. (1998) Two-Dimensional Phase Unwrapping: Theory, Algorithms, and Software. WileyInterscience, New York, USA.

44. Seed, G. (2000) Strength of Materials. Saxe Coburg Publications, Edinburgh, United Kingdom.

45. Rao, S. S. (2011) Mechanical Vibrations. Prentice Hall, Pearson, Upper Saddle River, New Jersey, USA.

46. Sol, H. (1986) Identification of anisotropic plate rigidities by vibration analysis. PhD thesis, Vrije Universiteit Brussel.

47. Lauwagie, T., Sol, H., Roebben, G., Heylen, W. and Shi, Y. (2002) Validation of the resonalyser method: an inverse method for material identification. in Proceedings of ISMA2002, pp. 687-694.

48. Devor, R., Chang, T. and Sutherland, J. (1992) Statistical Quality Design and Control: Contemporary Concepts and Methods. MacMillan, New York. 